\title{
DIAGNOSIS OF CONGENITAL ANEURYSM OF THE VENTRICULAR SEPTUM DURING LIFE
}

\author{
BY \\ ISRAEL STEINBERG * \\ From the Departments of Medicine and Radiology, The New York Hospital, Cornell Medical Centre
}

Received December 6, 1955

Aneurysms of the membranous portion of the ventricular septum are uncommon and there is general agreement that they are of congenital origin. These aneurysms may occur alone or with other cardiovascular anomalies. They are usually incidental findings at autopsy and seldom produce clinical manifestations. However, there are two reports of serious cardiac arrhythmias of many years duration that were attributed to the presence of septal aneurysms (Rogers et al., 1952; and Clark and White, 1952).

The demonstration of a localized bulge of the left ventricular septum in a patient studied by angiocardiography is herein reported. Even though autopsy confirmation of a ventricular septal aneurysm is lacking, the distinctive features of the angiocardiogram strongly favour such a diagnosis. Further evidence for this conclusion is provided by comparison with a membranous ventricular septal aneurysm recently noted as an incidental finding at autopsy. The purpose of this report is to describe the angiocardiographic appearance of a suspected ventricular septal aneurysm and to propose criteria for the diagnosis of such aneurysms during life.

\section{CASE REPORT}

A 60-year-old white housewife has been observed in the clinic and hospital for nearly five years. Cystitis was found on her first visit during 1950 and treated successfully. Subsequently she complained of mild dyspnoea and a routine chest roentgenogram revealed infiltration in the first right anterior interspace and an increase in the right hilar shadows. The heart appeared to be somewhat enlarged (Fig. 1A). Increased pulsation of the right descending pulmonary arterial branches was noted on fluoroscopic examination and an angiocardiogram was requested. Angiocardiography was normal except for a localized bulge to the right of the ventricular septum (Fig. 2).

During the five years under observation, the patient has had many complaints consisting of epigastric pain and discomfort. Studies, of the biliary, gastro-intestinal, and urinary systems revealed no abnormalities. The blood pressure was normal, 130/80. Standard, præcordial and vector electrocardiograms were normal except for left axis deviation and a P-R II of $0 \cdot 21$ sec. The heart examination was normal; no murmurs were elicited; the rhythm was normal sinus. Studies of the blood including the serologic test for syphilis were also normal. Tuberculin skin tests in all dilutions were negative. Biopsy of two skin papules failed to show sarcoidosis and no cervical, axillary, or inguinal masses have appeared. Roentgenograms of the chest during the five years have shown no change in appearance of the lungs, heart, or hilar structures.

\section{Discussion}

The embryological development of the membranous ventricular septum has been reviewed by Lev and Saphir $(1936,1938)$ and Rogers et al. (1952). The membranous ventricular septum is in part derived from endocardial cushion tissue and is intimately related to the closure of the atrioventricular canal. The closure is concerned with the union of tissues from the dorsal and ventral endocardial cushion systems, the primary interatrial septum, and the muscular interventricular

* Aided by a Grant-in-Aid from the American Cancer Society and the Mallinckrodt Chemical Works. 


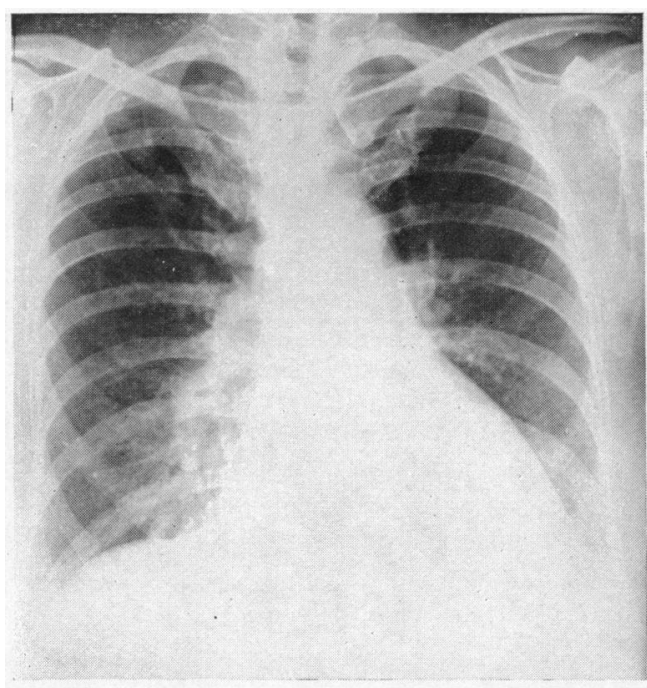

A

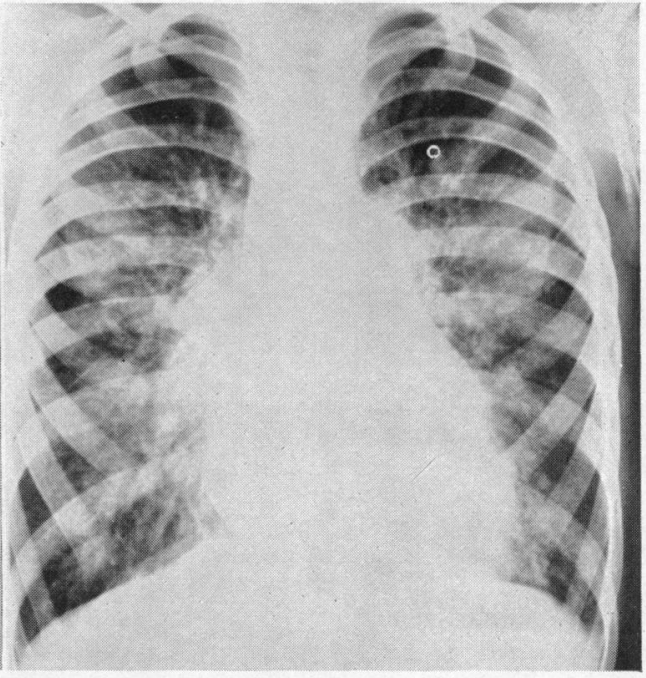

B

FIG. 1.-(A) Teleradiogram from a woman, aged 60, showing slight increase in the size of the heart and prominence of the descending right pulmonary arterial branches. There is also minor right infraclavicular pulmonary infiltration. (B) Teleradiogram from an 8-year-old boy who died of bronchiectasis, bronchopneumonia, pulmonary emphysema, and cor pulmonale associated with fibrocystic disease of the pancreas. Note the enlarged heart, prominent pulmonary artery, and diffuse pulmonary fibrosis.

septum. Lev and Saphir also established that Mall's (1912) explanation for the congenital theory of origin of membranous ventricular septal aneurysms was correct. They pointed out that it was due to an anomalous position of the aorta which displaced the membranous septum into a horizontal position to form a mild transposition. Accordingly, ventricular septal aneurysms may occur as single lesions or in association with anomalies of the aorta and interatrial and interventricular septal defects (Brown, 1950).

About 80 cases of congenital aneurysm of the membranous ventricular septum have been reported. Lev and Saphir (1938) recorded 70 previously reported cases and added two new ones. Castoldi (1942), Leckert and Sternberg (1950), Vanecek (1950), Schlicter et al. (1954), Kjellberg et al. (1955), and Clark and White (1952) added a case; while Rogers et al. (1952), added two new ones. As for incidence, Rae (1936) noted that four membranous ventricular septal aneurysms were found in 3000 autopsies ; on the other hand, only two were found in a total of 16,000 autopsies in this institution.

Fig. 3 is an autopsy specimen, showing an aneurysm of the membranous portion of the ventricular septum-an incidental finding in an eight-year-old boy. Death was due to bronchiectasis, broncho-pneumonia, pulmonary emphysema, and chronic cor pulmonale that resulted from long-standing pulmonary disease associated with fibrocystic disease of the pancreas (Fig. 1B). The aneurysm consisted of a one-cm. pouch which bulged from the membranous part of the interventricular septum into the right ventricle and was attached to a tricuspid valve leaflet. The heart weighed $180 \mathrm{~g}$. (expected weight $110 \mathrm{~g}$.). The right atrium and ventricle were much dilated; the right ventricle was hypertrophied and measured $8 \mathrm{~mm}$. The pulmonary artery was conspicuously dilated. The left atrium and ventricle were normal; the left ventricle wall measured $8 \mathrm{~mm}$. No communication between the ventricles was noted and the fossa ovalis was closed. All the valves were thin, smooth, and intact, and had normal measurements; the coronary arteries contained no lesions.

Two years before his death he had been admitted in severe congestive heart failure, due to cor pulmonale secondary to pulmonary fibrosis, which responded to digitalis and organic mercurial 


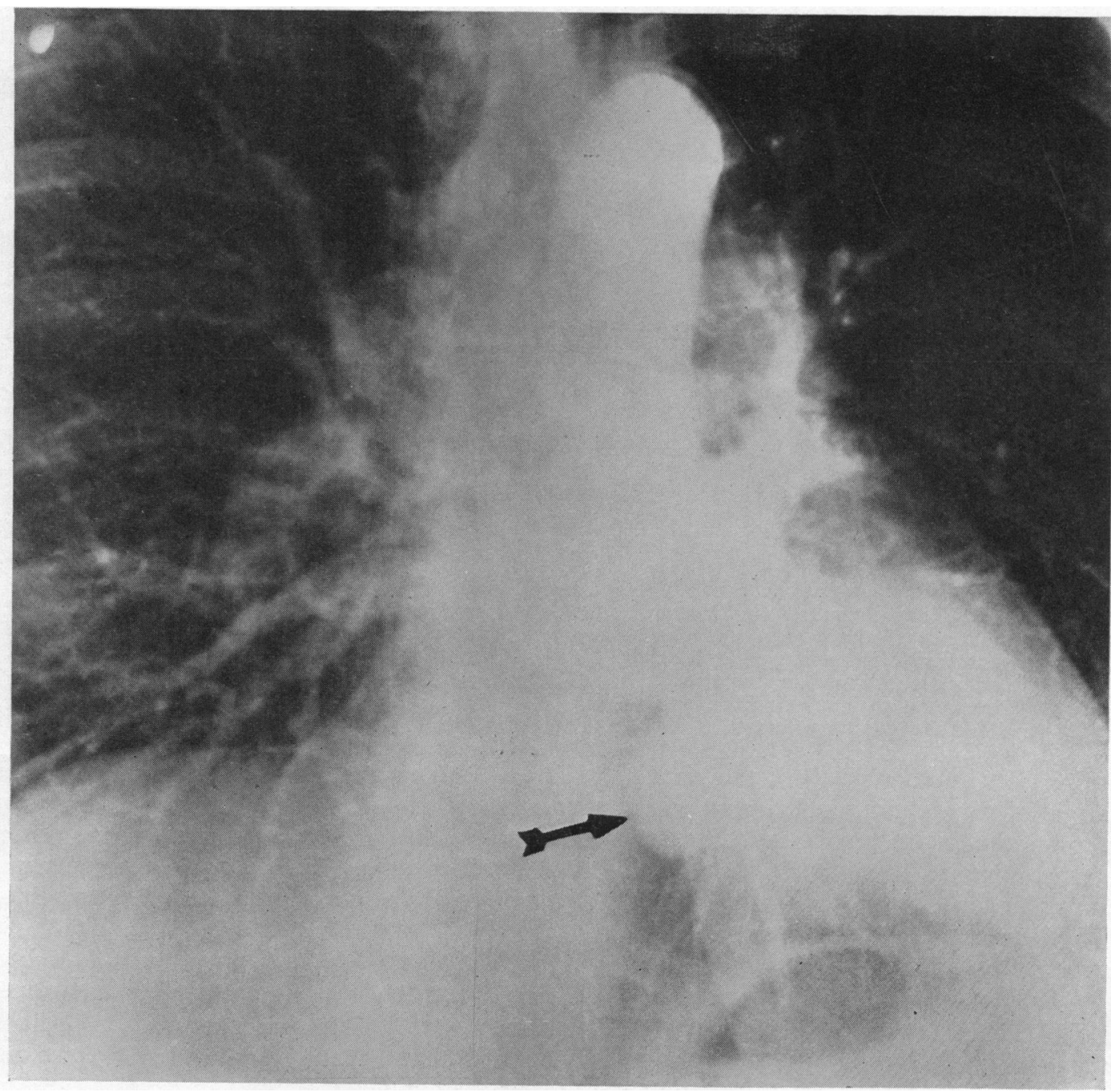

FIG. 2.-Frontal angiocardiogram (36-in. target film distance) shows normal opacification of the pulmonary veins, left ventricle, and aorta. Note the ventricular septal aneurysm (arrow).

therapy. During the two years under observation no cardiac murmurs were heard. The cardiogram showed right ventricular hypertrophy and right axis deviation.

In our living patient overt evidence of heart disease had not appeared during five years observation. Sarcoidosis was suspected at first because of the negative tuberculin tests and fullness of the right hilum which suggested mediastinal adenopathy, but skin biopsies failed to establish the presence of such a lesion and eye, lymph node, bone, kidney, or liver manifestations of sarcoidosis were never found. Bates and Walsh (1948) and Stephen (1954) reported sudden death of patients with sarcoidosis who had generalized sarcoidosis with myocardial and interventricular septal involvement at autopsy. The long survival period and absence of cardiac pain makes it unlikely that our patient has a septal aneurysmal due to sarcoid involvement of the heart.

As a rule, aneurysms of the membranous portion of the ventricular septum are of no great clinical importance. However, Rogers et al. (1952) point out that review of reported cases discloses that septal aneurysms may be sites of thrombus formation, superimposed bacterial endocarditis, and 
perforation. They may also be factors in the development of subaortic stenosis (Rae, 1936) and aortic insufficienty (Leckert and Sternberg 1950).

Serious cardiac arrhythmias presumably due to ventricular septal aneurysms have been reported in two patients. Rogers et al. (1952) described a case with extreme cardiac irritability of ten years duration: during life, there were attacks of auricular fibrillation, A-V nodal rhythm, paroxysmal auricular tachycardia, sinus tachycardia, and idioventricular rhythm; clinical evidences of myo-

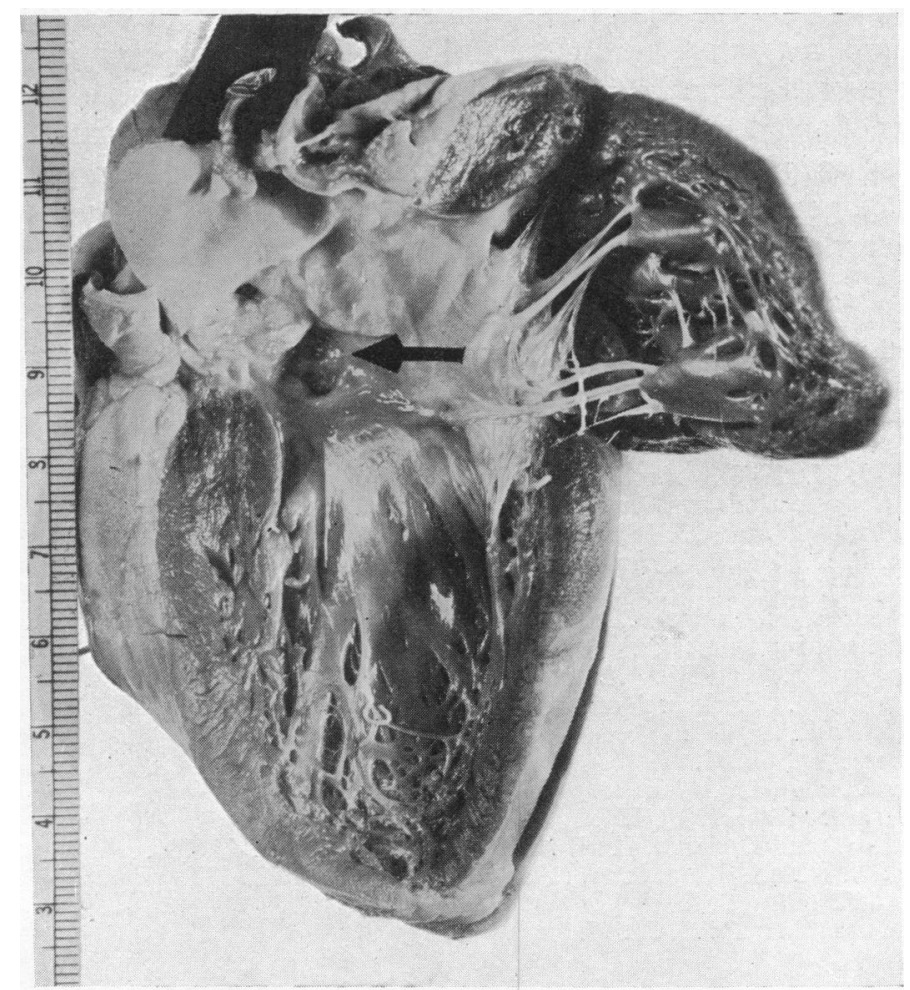

FIG. 3.-Incidental aneurysm of ventricular septum found in an 8-year-old boy. Aneurysm of the membranous portion of the left ventricular septum is located below the aortic cusps (arrow).

cardial infarction was present four years prior to death, and post mortem, in addition to a septal aneurysm, there was also a small ventricular septal infarction. Clark and White's (1952) patient had an aneurysm of the ventricular septum as the sole significant finding at autopsy: there was evidence of A-V block for 26 years prior to death, at first partial and later complete; death at the age of 47 was due to a series of Stokes-Adams attacks which were set off by paroxysmal ventricular flutter followed by ventricular tachycardia, ventricular standstill and complete A-V dissociation. Of interest, was the absence of bundle of His tissue in the carefully studied block sections of the entire aneurysm; histologically the aneurysm was composed of acellular collagenous tissue only.

The angiocardiographic demonstration of a localized bulge high in the left ventricular septum extending towards the right ventricular cavity is striking (Fig. 2). A filling defect of the right ventricle corresponding to the aneurysm was not recognized when the right ventricle was visualized. This may be because of the frontal positioning of the patient for angiocardiography. Had abnormality of the ventricular septum been anticipated, the left anterior oblique projection would have 
been selected and would have provided a true lateral view of the ventricles and septum (Dotter and Steinberg, 1951). The characteristic appearance of the localized bulge of the left ventricular septum in a patient relatively free of disease of the heart is believed to be strong evidence for the presence of an isolated ventricular septal aneurysm. Such a lesion has long been known to occur without clinical manifestations and has often, as in this case, been an incidental finding.

\section{SUMMARY}

A localized bulge high in the ventricular septum towards the right ventricle was recognized in the angiocardiogram of a woman, aged 60 , who had a slightly prolonged $P-R$ interval on electrocardiography and who was otherwise free of heart disease. On the basis of the angiocardiographic finding the diagnosis of an aneurysm of the membranous ventricular septum during life is proposed.

Although autopsy proof of the presence of the ventricular septal aneurysm is not available, the angiocardiographic diagnosis in this case seems warranted. The occasional occurrence of serious unexplained cardiac arrhythmias makes the diagnosis of ventricular septal aneurysm of more than academic interest.

Thanks are due to the Department of Pathology for permission to report the post-mortem findings in Case 2.

\section{REFERENCES}

Bates, G. S., and Walsh, J. M. (1948). Ann. intern. Med., 29, 306.

Brown, J. W. (1950). Congenital Heart Disease. Staples Press, London, p. 158.

Castoldi, P. (1942). Clin. Med. Ital., 73, 161.

Clark, R. J., and White, P. D. (1952). Circulation, 5, 725.

Dotter, C. T., and Steinberg, I. (1951). Angiocardiography. Paul B. Hoeber, Inc., New York.

Kjellberg, S. E., Mannheimer, E., Rudhe, J., and Jonsson, B. (1955). Diagnosis of Congenital Heart Disease. The Year Book Publishers, Inc., Chicago.

Leckert, J. T., and Sternberg, S. S. (1950). Amer. Heart J., 39, 768.

Lev. M., and Saphir, O. (1936). J. Tech. Methods, 16, 61.

L (1938). Arch. Path., 25, 819.

Mall, F. P. (1912). Anat. Rec., 6, 291.

(1912-13). Amer. J. Anat., 13, 249.

Rae, M. V. (1936). J. Tech. Methods, 15, 136.

Rogers, H. M., Evans, I. C., and Domeier, L. H. (1952). Amer. Heart J., 43, 781.

Schlicter, J., Hellerstein, H. K., and Katz, L. N. (1954). Medicine, 33, 43.

Stephen, J. D. (1954). Circulation, 9, 886.

Vanecek, E. (1950). Lékar. Listy, Brno., 5, 597. 